\title{
Expressional alterations in functional ultra-conserved non-coding rnas in response to all-trans retinoic acid - induced differentiation in neuroblastoma cells
}

Karen M Watters ${ }^{1,2^{*}}$, Kenneth Bryan ${ }^{1,2}$, Niamh H Foley ${ }^{1,2}$, Maria Meehan ${ }^{1,2}$ and Raymond L Stallings $s^{1,2}$

\begin{abstract}
Background: Ultra-conserved regions (UCRs) are segments of the genome ( $\geq 200 \mathrm{bp}$ ) that exhibit 100\% DNA sequence conservation between human, mouse and rat. Transcribed UCRs (T-UCRs) have been shown to be differentially expressed in cancers versus normal tissue, indicating a possible role in carcinogenesis. All-trans-retinoic acid (ATRA) causes some neuroblastoma (NB) cell lines to undergo differentiation and leads to a significant decrease in the oncogenic transcription factor MYCN. Here, we examine the impact of ATRA treatment on T-UCR expression and investigate the biological significance of these changes.

Methods: We designed a custom tiling microarray to profile the expression of 481 T-UCRs in sense and anti-sense orientation (962 potential transcripts) in untreated and ATRA-treated neuroblastoma cell lines (SH-SY5Y, SK-N-BE, LAN-5). Following identification of significantly differentially expressed T-UCRs, we carried out siRNA knockdown and gene expression microarray analysis to investigate putative functional roles for selected T-UCRs.

Results: Following ATRA-induced differentiation, 32 T-UCRs were differentially expressed (16 up-regulated, 16 down-regulated) across all three cell lines. Further insight into the possible role of T-UC.300A, an independent transcript whose expression is down-regulated following ATRA was achieved by siRNA knockdown, resulting in the decreased viability and invasiveness of ATRA-responsive cell lines. Gene expression microarray analysis following knockdown of T-UC.300A revealed a number of genes whose expression was altered by changing T-UC.300A levels and that might play a role in the increased proliferation and invasion of NB cells prior to ATRA-treatment.

Conclusions: Our results indicate that significant numbers of T-UCRs have altered expression levels in response to ATRA. While the precise roles that T-UCRs might play in cancer or in normal development are largely unknown and an important area for future study, our findings strongly indicate that the function of non-coding RNA T-UC.300A is connected with proliferation, invasion and the inhibition of differentiation of neuroblastoma cell lines prior to ATRA treatment.
\end{abstract}

Keywords: ATRA, neuroblastoma, Transcribed ultra-conserved regions, Differentiation

\footnotetext{
*Correspondence: kwatters@bsd.uchicago.edu

${ }^{1}$ Cancer Genetics, Department of Molecular \& Cellular Therapeutics, Royal College of Surgeons in Ireland, Dublin, Ireland

${ }^{2}$ Children's Research Centre, Our Lady's Children's Hospital Crumlin, Dublin, Ireland
}

\section{Biomed Central}

(C) 2013 Watters et al.; licensee BioMed Central Ltd. This is an Open Access article distributed under the terms of the Creative Commons Attribution License (http://creativecommons.org/licenses/by/2.0), which permits unrestricted use, distribution, and reproduction in any medium, provided the original work is properly cited. 


\section{Background}

Neuroblastoma (NB) is a highly heterogenous childhood cancer that arises from precursor cells of the sympathetic nervous system [1]. Clinical behaviour of these tumors, ranging from spontaneous regression to rapid progression and death due to disease, is highly correlated with a number of genomic alterations involving ploidy, MYCN amplification (MNA), and large-scale genomic imbalances such as loss of chromosome 1p, 3p, 11q and gain of 17q. MNA and loss of heterozygosity on chromosome 11q are particularly associated with aggressive disease course and represent independent genetic subtypes of NB. Each genetic subtype of NB, such as MNA or 11q-, also has significant differences in the expression patterns of large sets of protein coding genes [2-6], and in the expression profiles of non-coding RNAs such as microRNAs [7-11].

Ultra-conserved regions (UCRs) are by definition DNA segments that are at least $200 \mathrm{bp}$ in length and that are $100 \%$ conserved between human, rat and mouse genomes. Four hundred and eighty-one such regions have been identified [12]. UCRs are comprised of three basic types intragenic (39\%), intronic (43\%) and exonic (15\%), which also includes 'partly exonic' and 'exon containing'. Approximately $3 \%$ of UCRs are not easily classified, due to their juxtaposition with alternative splice variants of host genes and the resulting variable annotation. Calin et al. [13] carried out the first analysis of transcribed UCRs (T-UCRs) in cancer, demonstrating that approximately 9\% of the 962 possible T-UCRs (sense + anti-sense) were aberrantly transcribed in either carcinomas or leukemias relative to normal tissue. Most significantly, the authors further demonstrated that siRNA-mediated down-regulation of one T-UCR (T-UC.73A) significantly increased apoptosis in a colorectal cancer cell line. Two recent studies have demonstrated that analysis of UCR expression signatures can also be applied to the evaluation of NB tumors $[14,15]$. Differential UCR expression profiles were shown to be associated with outcome in short-term versus long-term survivors with high-risk, stage $4 \mathrm{NB}$ [15]. In addition, Mestdagh et al., found an expression signature of upregulated T-UCRs in MNA compared to non-MNA tumors [14].

The synthetic retinoic acid, 13-cis-retinoic acid, is an established component of the treatment given to children with high-risk NB to reduce minimal residual disease $[16,17]$ and exposure of a number of NB cell lines, such as SK-N-BE, to ATRA induces neural cell differentiation along with down-regulation of MYCN [18]. Here, we identify T-UCRs that are responsive to the retinoid, all-transretinoic acid (ATRA), across three ATRA-sensitive cell lines and investigate the functional role of the deregulated transcript T-UC.300A.

Previous studies analyzing UCR expression in NB have used qPCR, involving reverse transcription with random primers, which is unable to distinguish between transcripts originating from the sense or the anti-sense genomic strand. Our approach involved the construction of tiling arrays for 962 UCR regions, allowing for the detection of both sense and anti-sense transcripts and for expression of host genes. We identified and validated a number of $\mathrm{T}$ UCRs that are differentially expressed following ATRAinduced differentiation. Further insight into a functional role for T-UC.300A was achieved by siRNA knockdown resulting in the decreased viability and invasiveness of ATRA-responsive cell lines. As T-UC.300A is downregulated following ATRA treatment, our findings strongly indicate that its function is connected with the increased proliferation and invasion of NB cells prior to ATRAtreatment.

\section{Methods}

\section{Cell culture}

The NB cell lines SK-N-BE and SH-SY5Y were obtained from the American Type Culture Collection (ATCC). Cell culture medium consisted of Ham's F12 and EMEM (50:50) supplemented with fetal bovine serum (10\%), nonessential amino acids $(0.5 \%)$, L-glutamine $(0.5 \%)$ and penicillin/streptomycin (1\%). The LAN-5 cell line was obtained from the Children's Oncology Group Repository. LAN-5 culture medium consisted of RPMI supplemented with penicillin/streptomycin (1\%). All cell culture reagents were obtained from GIBCO.

\section{ATRA treatment}

All-trans retinoic acid (ATRA) was administered daily (5 $\mu \mathrm{M}$ final conc.) to cells over a period of 7 days. Treated and untreated cells were fixed using $4 \%$ paraformaldhyde (Sigma) and permeabilised in $0.5 \%$ Triton X-100. Cells were probed using the neuronal marker BIII Tubulin (Abcam), and subsequently were incubated with the flourescein-conjugated goat anti-rabbit Alexa Flour 488 antibody (Invitrogen). Cells were washed in PBS and then counterstained using DAPI. The Nikon TE2000s Fluorescence microscope was used to examine the cells and photographs were taken with the Hamamatsu (Orca 285) CCD Camera.

\section{Microarray design}

The UCR custom tiling microarray was developed using Roche NimbleGens $4 \times 72 \mathrm{~K}$ array, composed of four identical subarrays, tiling 962 ultra-conserved sequences (481 sequences in both sense and anti-sense orientation). Oligo lengths ranged from $50 \mathrm{mer}$ to $72 \mathrm{mer}$, in order to maintain a similar Tm across all probes. The genomic coordinates of the 481 UCRs (Build HG17, May 2004) were obtained from http://users.soe.ucsc.edu/ jill/ultra. html and converted to Build HG18, using UCSC's Batch Coordinate Conversion tool (http://genome.ucsc.edu/ 
cgi-bin/hgLiftOver). In addition to the ultra-conserved sequences themselves, tiling coverage also spanned 2500 bases upstream and 500 bases downstream of the location of each UCR on both sense and anti-sense strands. Probes were designed in two separate containers (sense and antisense) on each subarray to facilitate independent data analysis. Array design is available in ArrayExpress- Accession number A-MEXP-1899.

For gene expression, the Homo Sapiens $4 \times 72 \mathrm{~K}$ gene expression array from Roche NimbleGen was used.

\section{Sample preparation}

The QIAGEN RNeasy Mini Kit (Cat. No. 74101) was used to extract RNA from cells (untreated at Day 0 and ATRAtreated at Day 7). DNase treatment was included to ensure complete removal of any genomic DNA that could affect results by also hybridising to the microarrays. RNA integrity was confirmed with the Agilent RNA Nano 6000 kit (Cat. No. 5067-1511) and an Agilent Bioanalyzer. Only RNA with an RNA Integrity Number (RIN) of $>8$ was used for microarray analysis. For tiling microarrays, doublestranded cDNA was synthesised from 3 ug total RNA using the ExpressArt TRinucleotide mRNA Amplification Micro Kit (AmpTec). In vitro transcription using the ExpressArt AminoAllyl Add-on Module (AmpTec) generated aminoallyl modified-anti-sense RNA (aRNA), which was subsequently incubated with NHS-Cy3 (Amersham). Following purification, $4 \mu \mathrm{g}$ Cy3-aRNA was hybridised to microarrays.

For gene expression microarray analysis following siRNA knockdown of T-UC.300A or ATRA treatment, sample preparation was carried out as previously described [19].

\section{RT-PCR}

Reverse transcription for transcribed UCRs was carried out on 1ug total RNA with gene-specific primers and the SuperScript III First-Strand Synthesis System for RT-PCR (Invitrogen) in a total reaction volume of $20 \mathrm{ul}$ (T-UC.324: 5' CCCCATCCCATATGACACTC 3'; T-UC .300A: $5^{\prime}$ AAAAGTGGAAATCAATTTTGAAGG $3^{\prime}$ ). Real-time PCR was carried out using custom designed TaqMan assays for T-UC.324 and T-UC.300A (Applied Biosystems).

\section{Western blot}

Total protein was isolated from cells using a radioimmunoprecipitation assay (RIPA) lysis buffer (Sigma). Cell pellets were washed with PBS and solubilized in RIPA for 30 mins. Protein concentration was measured using the BCA assay from Pierce. Proteins were fractionized on $6 \%$ or $10 \%$ polyacrylamide gels, and blotted onto nitrocellulose membrane. MYCN protein and the neuronal marker $\beta$ - III Tubulin were detected by Western Blot using the mouse monoclonal antibody SC-
53993 (Santa Cruz) and the rabbit polyclonal antibody AB-8191 (Abcam) respectively.

\section{siRNA knockdown}

siRNAs against T-UC.324 and T-UC.300A were designed using Dharmacons siRNA Design Centre. siRNAs were as follows:

\section{T-UC.324: TTACCTAACCAGTGATTAA}

(sense strand sequence)

T-UC.300A: ATTCATGGATGGAGATTGA

(sense strand sequence)

Cells were transfected with T-UCR siRNAs (final concentration $50 \mathrm{nM}$ ) or negative control siRNA (Dharmacon Negative Control \#1, final concentration $50 \mathrm{nM}$ ) using the transfection reagent Lipofectamine (Invitrogen). Media was changed after $24 \mathrm{hrs}$. RNA was extracted $120 \mathrm{hrs}$ after transfection.

\section{Acid phosphatase assay}

Cells were transfected with siRNAs in 96-well plates using Lipofectamine, and plates were set up for timepoints 24120 hrs. At each timepoint, the appropriate plate was washed twice with PBS. $10 \mathrm{mM}$-nitrophenol phosphate in $0.1 \mathrm{M}$ sodium acetate with $0.1 \%$ triton $\mathrm{X}-100$ was added. Plates were incubated at $37^{\circ} \mathrm{C}$ for two hours and the reaction was stopped with $50 \mu \mathrm{L} 1 \mathrm{M}$ sodium hydroxide per well. Absorbance was measured at $405 \mathrm{~nm}$ using the Victor X3 Multi-Label Reader (Perkin Elmer).

\section{Cell proliferation assay}

The effect of siRNA knockdown of T-UC.300A on the rate of cell proliferation in SH-SY5Y cells was assayed using the Cell Proliferation ELISA, BrdU (colorimetric) kit (Roche, Cat. No. 11647229 001). Cells were transfected with siRNA against T-UC.300A or a scrambled control and were cultured in a tissue culture grade, flat bottom 96-well plate for 96 hours $(n=4)$. BrdU labeling solution was added to each well (final concentration $10 \mu \mathrm{M}$ BrdU) and the cells were re-incubated for an additional 2 hours. The labeling medium was then removed and the cells were fixed and denatured. Denatured DNA was incubated with an anti-BrdU monoclonal antibody conjugated with peroxidase for 90 minutes, followed by washing. $100 \mu \mathrm{l} /$ well substrate solution was added and the plate was incubated at +15 to $+25^{\circ} \mathrm{C}$ for 5 minutes. Stop solution $(25 \mu \mathrm{l}$ $1 \mathrm{M} \mathrm{H}_{2} \mathrm{SO}_{4}$ ) was added to each well and mixed thoroughly. Absorbance of samples was measured using the Victor X3 Multi-Label Reader (Perkin Elmer) at $450 \mathrm{~nm}$, reference wavelength $690 \mathrm{~nm}$. Following subtraction of the absorbance value for the blank control from all readings, the change in proliferation rate induced by knockdown of TUC.300A was determined. 


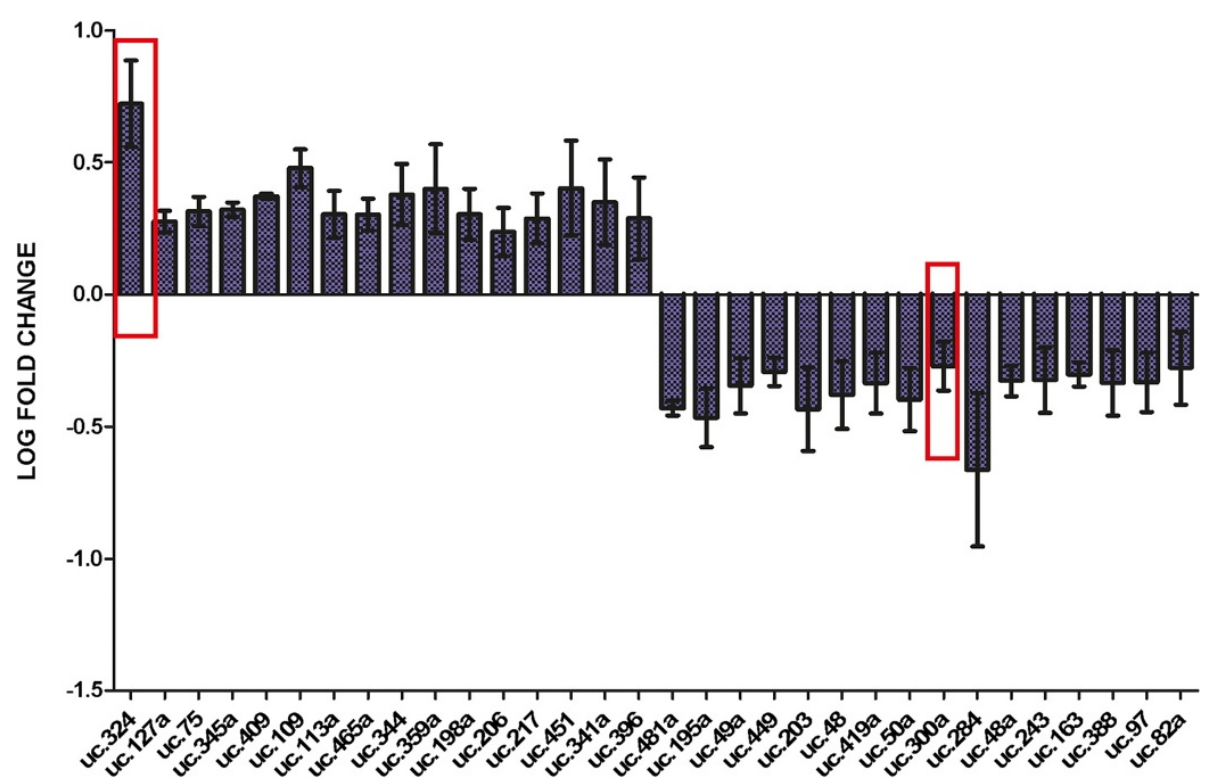

Figure 1 T-UCRs expressed following ATRA-treatment. Thirty-two T-UCRs were differentially expressed following ATRA-induced differentiation of neuroblastoma cell lines with $p<0.05$, with 16 transcripts up-regulated and 16 down-regulated greater than 1.5 fold in at least $4 / 6$ samples. The red boxes indicate the two T-UCRs selected for PCR validation and functional analysis.

\section{Invasion assays}

Cells were transfected in a 6-well plate. 48 hours after transfection, cells were trypsinized and counted. $2.5 \times 10^{4}$ cells were added to BD BioCoat ${ }^{\text {TM }}$ Growth Factor Reduced MATRIGEL ${ }^{\mathrm{TM}}$ Invasion Chambers (BD Biosciences) as per manufacturers' instructions and incubated for a further 72 hrs. To determine the average number of invading cells, MATRIGEL inserts were stained with crystal violet and viewed under the microscope. The number of cells/ field, in 5 random fields were counted at $200 \times$ magnification. Mean values of duplicate experiments were calculated and results subjected to t-test.

\section{Data processing and bioinformatics analysis Gene expression arrays}

The mRNA expression data was analysed using NimbleGens NimbleScan software version 2.4, which applied quantile normalization to the data [20] and expression values were obtained using the Robust Multi-Chip Average algorithm as described by Irizarry et al. [21]. Expressional alterations of 1.5 fold across both biological repeats were considered significant.

\section{T-UCR tiling arrays}

Microarrays were scanned using the GenePix 4000B scanner. Pair reports were generated using NimbleGen's NimbleScan software version 2.4, and normalised by applying quantile normalisation. Median smoothing was first carried out on both the forward and reverse T-UCR tiling arrays with a window of 3 probes to remove outlying probe values. T-UCRs were considered expressed if their mean probe value was above the array background (median expression of the array). Only T-UCRs that were expressed in all 3 cell lines were considered. T-UCRs that were differentially expressed at least 1.5 fold in 2 out of 3 cell lines $(n \geq 4)$ were selected. For these selected T-UCRs, $\mathrm{p}$-values were calculated across all 3 cell lines $(\mathrm{n}=6$ biological samples) using Student's t-test, and only T-UCRs with a p-value $<0.05$ were considered significant.

\section{Correlation of intragenic $t$-ucrs and host gene expression}

Using the expression data from the ATRA-treated and untreated tiling arrays, intragenic T-UCRs transcribed in the same directions as their host genes were assessed for independent transcription relative to exonic expression, if exons were covered by the tiled region. Pearson's correlation of T-UCR expression (mean probe value) and gene (mean expression of exonic probes) over experimental samples was used to assess T-UCR/Host gene expression correlation. Significance of this correlation was determined from a table of critical values for Pearson's Correlation over various degrees of freedom.

\section{Cluster analysis of intragenic $t$-ucrs expression}

T-UCR expression was measured on the forward and reverse strand however this causes problems when calculating object similarity in cluster analyses i.e. every object will be represented twice in the expression dataset by two independent features sets, one on the forward strand and one on the reverse strand. The matrix was 
Table 1 T-UCRs altered by ATRA

\begin{tabular}{|c|c|c|c|c|c|c|c|c|c|}
\hline T-UCR name & $\begin{array}{c}\text { SK-N-BE } \\
(n=2)\end{array}$ & $\begin{array}{l}\text { LAN-5 } \\
(n=2)\end{array}$ & $\begin{array}{l}\text { SH-SY5Y } \\
(n=2)\end{array}$ & Type & Orientation with host gene & Host gene & UCR_host correlation & Previously reported data & $\mathrm{p}$-value \\
\hline uc.324 & 5.57 & 2.68 & 9.86 & Exon & anti-sense & MPPED2 & & & 0.0019 \\
\hline uc.127a & 2.02 & 2.12 & 1.57 & Intergenic & 0 & 0 & & & 0.0026 \\
\hline uc.75 & 1.86 & 2.66 & 1.77 & Exon & anti-sense & ZEB2 & & & 0.0030 \\
\hline uc.345a & 2.02 & 2.37 & 1.92 & Intron & anti-sense & $\mathrm{HOXC4}$ & & & 0.0066 \\
\hline uc.409 & 2.29 & 2.45 & 2.33 & Intergenic & 0 & 0 & & FRA<2MB (Calin et al., 2007) & 0.0107 \\
\hline uc.109 & 3.58 & 2.16 & 3.52 & Intron & anti-sense & LOC375295 & & HOX cluster (Calin et al., 2007) & 0.0123 \\
\hline uc.113a & 2.46 & 2.48 & 1.33 & Intergenic & 0 & 0 & & & 0.0134 \\
\hline uc.465a & 2.62 & 1.89 & 1.63 & Intron & anti-sense & POLA1 & & & 0.0154 \\
\hline uc.344 & 1.41 & 2.86 & 3.38 & Intron & sense & $\mathrm{HOXC4}$ & Sig. correlated & FRA<2MB (Calin et al., 2007) & 0.0156 \\
\hline uc.359a & 5.08 & 2.35 & 1.33 & Exon & sense & NOVA1 & Sig. correlated & & 0.0194 \\
\hline uc.198a & 1.31 & 2.25 & 2.76 & Intergenic & 0 & 0 & & & 0.0204 \\
\hline uc.206 & 1.14 & 2.01 & 2.24 & Intergenic & 0 & 0 & & & 0.0245 \\
\hline uc. 217 & 1.25 & 2.39 & 2.44 & Exon & sense & VSTM2A & Sig. correlated & & 0.0256 \\
\hline uc.451 & 1.15 & 3.01 & 4.67 & Intron & anti-sense & TSHZ3 & & & 0.0297 \\
\hline uc.341a & 1.28 & 4.54 & 1.92 & Exon & anti-sense & HOXC10 & & FRA $<2 M B$ (Calin et al., 2007) & 0.0303 \\
\hline uc.396 & 1.11 & 3.77 & 1.75 & Intergenic & 0 & 0 & & Down-regulated in HCC (Calin et al., 2007) & 0.0346 \\
\hline uc.481a & 0.38 & 0.41 & 0.33 & Exon & anti-sense & STAG2 & & & 0.0005 \\
\hline uc.195a & 0.50 & 0.38 & 0.21 & Intron & sense & C6orf167 & No data available & & 0.0013 \\
\hline uc.49a & 0.48 & 0.66 & 0.29 & Exon & sense & FAM98A & No data available & $\begin{array}{l}\text { Inferred role in differentiation for UCR49 } \\
\text { (Mestdagh et al., 2010) }\end{array}$ & 0.0082 \\
\hline uc.449 & 0.42 & 0.49 & 0.64 & Intron & sense & ZNF536 & No data available & & 0.0086 \\
\hline uc.203 & 0.18 & 0.47 & 0.59 & Exon & sense & QKI & Sig. correlated & LOH (Calin et al., 2007) & 0.0097 \\
\hline uc.48 & 0.31 & 0.75 & 0.31 & Exon & anti-sense & PUM2 & & & 0.0109 \\
\hline uc.419a & 0.35 & 0.78 & 0.36 & Exon & sense & SFRS1 & Not Correlated & FRA<2MB, HPV16<2.5MB (Calin et al., 2007) & 0.0125 \\
\hline uc.50a & 0.35 & 0.68 & 0.27 & Intron & sense & SFRS7 & Sig. correlated & & 0.0131 \\
\hline uc.300a & 0.38 & 0.51 & 0.79 & Intron & anti-sense & PAX2 & & $\begin{array}{l}\text { LOH, HOX gene, Up-regulated in CRC } \\
\text { (Calin et al., 2007) }\end{array}$ & 0.0135 \\
\hline
\end{tabular}


Table 1 T-UCRs altered by ATRA (Continued)

\begin{tabular}{|c|c|c|c|c|c|c|c|c|c|}
\hline uc.284 & 0.81 & 0.09 & 0.14 & Intergenic & 0 & 0 & & & 0.0138 \\
\hline uc.48a & 0.37 & 0.59 & 0.48 & Exon & sense & PUM2 & No data available & & 0.0160 \\
\hline uc.243 & 0.59 & 0.27 & 0.67 & Intron & sense & ZFHX4 & Not correlated & AMPLIF (Calin et al., 2007) & 0.0176 \\
\hline uc.163 & 0.59 & 0.41 & 0.51 & Intergenic & 0 & 0 & & & 0.0179 \\
\hline uc.388 & 0.28 & 0.75 & 0.47 & Intron & sense & TCF12 & No data available & $\begin{array}{l}\text { Up-regulated in CRC (Calin et al., 2007) } \\
\text { Down-regulated in CRC (Sana et al., 2012) }\end{array}$ & 0.0202 \\
\hline uc.97 & 0.55 & 0.65 & 0.28 & Intron & sense & HAT1 & Not correlated & HOX gene (Calin et al., 2007) & 0.0307 \\
\hline uc.82a & 0.40 & 0.37 & 0.99 & Intergenic & 0 & 0 & & & 0.0499 \\
\hline
\end{tabular}

Table shows the mean fold change across two samples for each cell line following ATRA treatment. 32 T-UCRs showed significantly altered expression following ATRA treatment across all samples ( $\mathrm{p}<0.05$ ). 


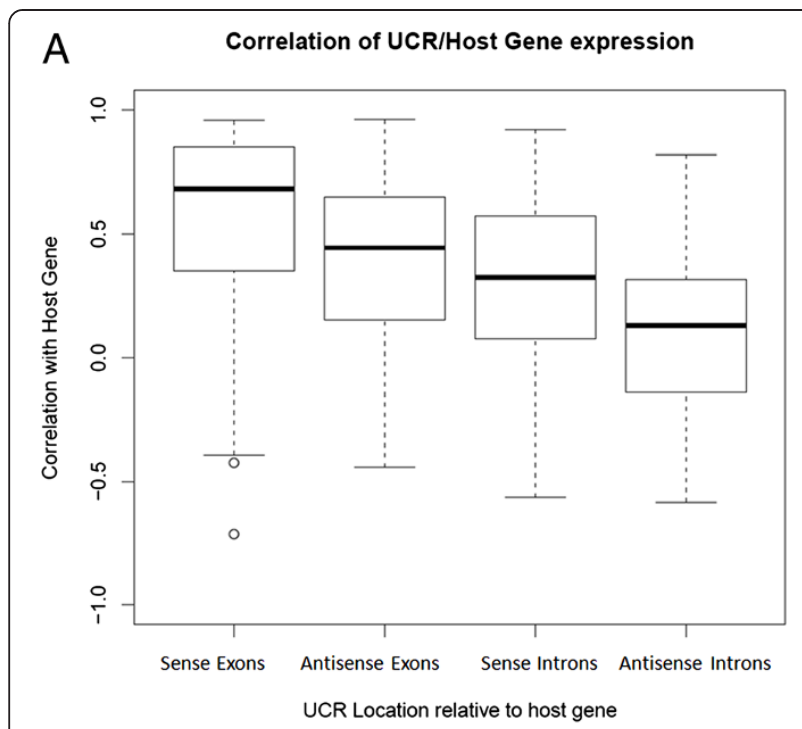

B

B Expression Anti-sense Intronic UCR 350 vs host DACH1

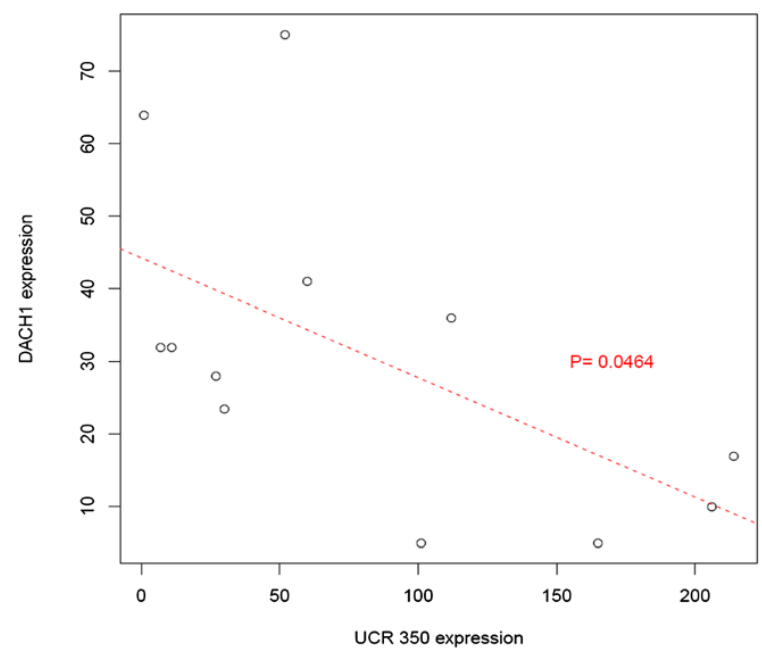

C

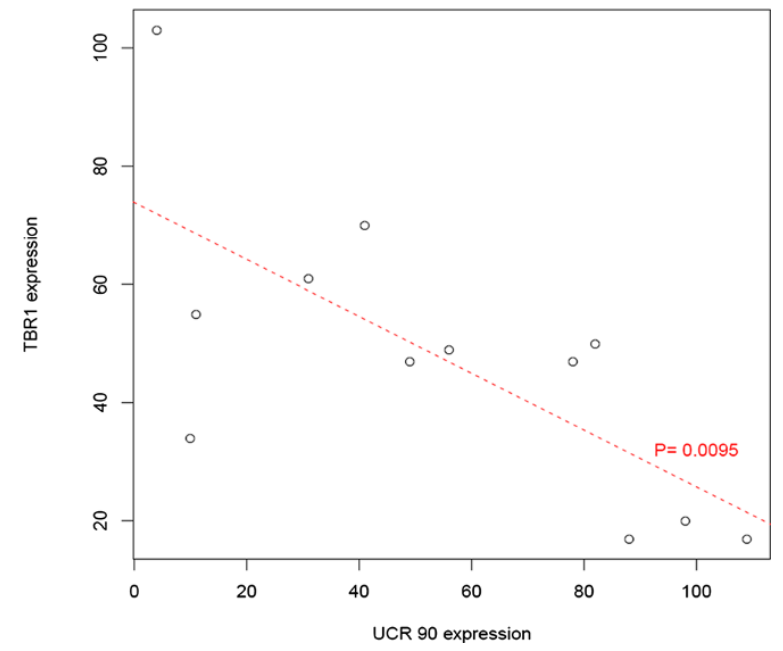

Figure 2 T-UCR-host gene correlation. Intragenic T-UCRs were assessed for correlative expression relative to host gene exonic expression, if exons were covered by the tiled region. (A) Box plots showing correlations between intragenic T-UCR expression and host gene expression. Anti-sense intronic T-UCRs displayed the lowest correlation with host genes, and sense exonic T-UCRs displayed the highest. The expression of anti-sense intronic T-UCRs (B) T-UC.350 and (C) T-UC.90 is significantly anti-correlated with the expression of their host genes DACH1 and TBR1 respectively $(p<0.05)$.

re-ordered by first re-orientating the arbitrary direction of T-UCR expression so that it was relative to the host gene with sense (S) referring to those T-UCRs transcribed with the host gene and anti-sense (A) referring to those T-UCRs transcribed in the opposite direction to the host gene. The $\mathrm{S}$ and $\mathrm{A}$ expressions for each T-UCR were then merged into a single feature set, thus the data matrix of 962 and $F$ expression features was converted into a data matrix of 481 T-UCRS and $2 F$ features $\left(F_{S}+F_{A}\right)$. Similarity of expression of intragenic T-UCRs was then determined using Spearman's rank correlation. Hierarchical clustering was subsequently carried out using Ward's metric and presented with accompanying heatmap visualization. Analysis was carried out using algorithms implemented in Java v1.6 and packages from R statistical programming language $\mathrm{R}$ version 2.8.0.

\section{Results}

In order to examine the expression levels of all T-UCRs, a custom array was designed tiling across all UCRs on both plus and minus genomic strands. Thus, a potential 962 T-UCRs, transcribed from 481 genomic regions when taking into account forward and reverse transcripts, could be detected. Tiling also incorporated 2500 bases upstream and 500 bases downstream of each UCR. Discernment of the genomic strand from which each TUCR originated was possible by synthesizing anti-sense RNA (aRNA), which is complementary to the original transcript, for $\mathrm{Cy} 3$ labelling and array hybridization.

\section{Differential expression of t-ucrs following atra treatment}

In order to identify putative T-UCRs involved in neuroblastoma cell differentiation, three ATRA-sensitive NB cell lines, SK-N-BE $(n=2)$, LAN-5 $(n=2)$ and SH-SY5Y $(\mathrm{n}=2)$, were treated daily with ATRA for 7 days, which resulted in extensive neurite outgrowth. Differentiation was also confirmed at protein level through the observed up-regulation of the differentiation marker TUBB3 and down-regulation of MYCN (Additional file 1: Figure S1). Expression analysis using the tiling arrays identified 32 transcripts whose expression was significantly altered $(\mathrm{p}<0.05)$ following ATRA-induced differentiation (fold change of $\geq 1.5$ in at least 4 out of the 6 biological repeats) (Figure 1, Table 1). The array results were 
A

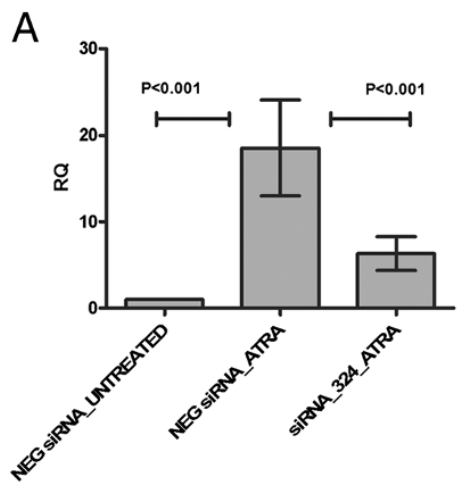

C

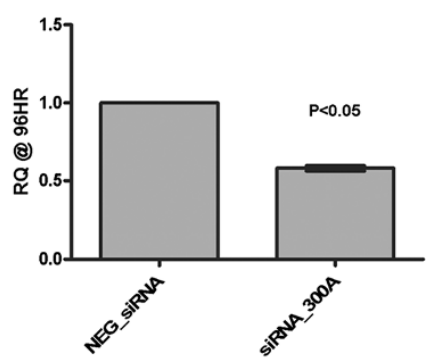

E

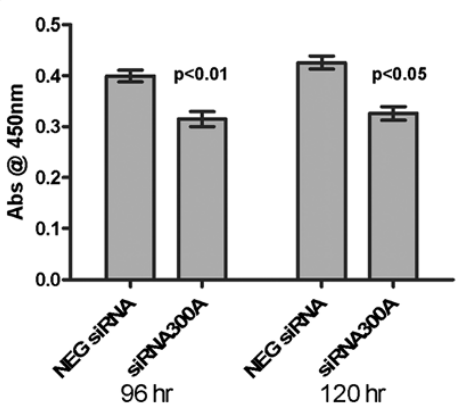

B

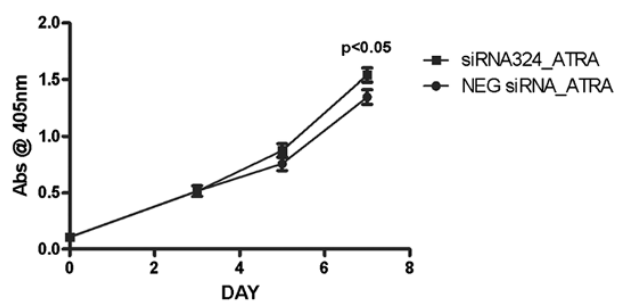

D

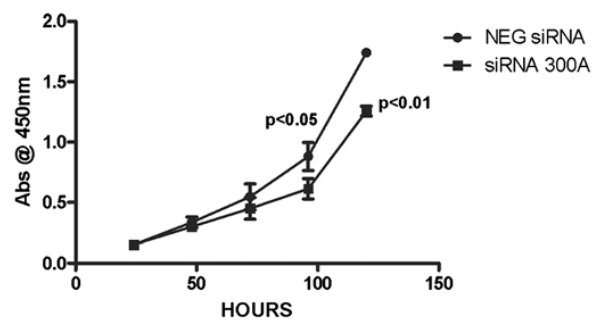

F

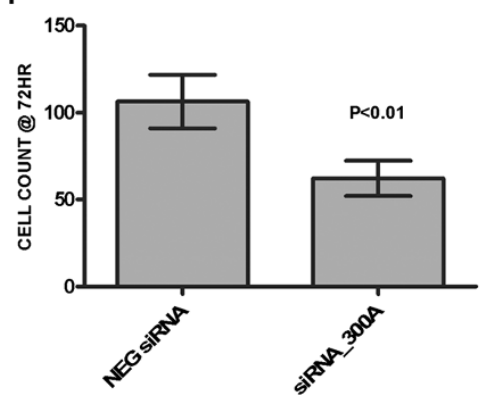

G

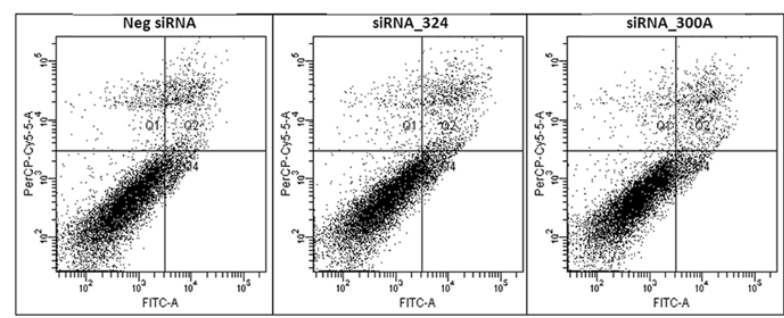

Figure 3 Functional analysis of T-UCRs. T-UC.324 and T-UC.300A transcripts were knocked down using custom designed siRNA (Dharmacon). (A) Knockdown of T-UC.324 significantly abrogated its up-regulation following ATRA-treatment, (B) but had no effect on cell viability. (C) A significant decrease in T-UC.300A levels was observed following siRNA transfection. (D) Knockdown of T-UC.300A resulted in a decrease in cell invasion as shown by MATRIGEL invasion assay $(p<0.01)$ and $(\mathbf{E})$ cell viability as shown by acid phosphatase assay $(P<0.01)$. (F) Reduction in cell viability following T-UC.300A knockdown was shown by BrdU assay to be due to a decrease in cell proliferation ( $p<0.05)$. (G) Knockdown of neither T-UC.324 nor T-UC.300A had any effect on apoptosis. 
Table 2 Top 15 up- and down-regulated protein coding genes following siRNA knockdown of T-UC.300A in SHSY5Y cells

\begin{tabular}{|c|c|c|}
\hline Gene name & $\begin{array}{c}\text { Mean fold change } \\
\text { T-UC.300A siRNA knockdown }\end{array}$ & $\begin{array}{c}\text { Mean fold } \\
\text { change after ATRA }\end{array}$ \\
\hline INPP5D & 3.72 & \\
\hline CALCA & 3.67 & 40.16 \\
\hline LOC221442 & 3.24 & \\
\hline LOC644242 & 3.23 & 5.28 \\
\hline ТТС9B & 2.94 & 2.18 \\
\hline GDF15 & 2.93 & 5.10 \\
\hline USP9X & 2.78 & \\
\hline COL1A2 & 2.75 & \\
\hline C10orf85 & 2.70 & \\
\hline FLJ4184 & 2.62 & \\
\hline HBE1 & 2.53 & \\
\hline DPF3 & 2.44 & \\
\hline LOC402199 & 2.43 & 37.41 \\
\hline FLJ44635 & 2.42 & 1.65 \\
\hline KLF2 & 2.38 & \\
\hline RAB27B & 0.35 & \\
\hline FLJ44124 & 0.37 & \\
\hline CNTNAP5 & 0.07 & \\
\hline PAX5 & 0.38 & \\
\hline INHBA & 0.39 & \\
\hline PCDHGA10 & 0.39 & \\
\hline TOR1AIP2 & 0.39 & 0.23 \\
\hline CHR4155SYT & 0.39 & \\
\hline ZNF718 & 0.40 & \\
\hline PRTG & 0.41 & \\
\hline HOOK1 & 0.41 & 0.38 \\
\hline LOC643647 & 0.42 & 0.59 \\
\hline LOC643523 & 0.42 & \\
\hline IPMK & 0.42 & \\
\hline ERCC4 & 0.43 & \\
\hline
\end{tabular}

Table shows fold change following knockdown across two biological repeats and also the corresponding mean fold change following ATRA if present.

validated by $\mathrm{qPCR}$ for selected T-UCRs using custom designed TaqMan assays (Additional file 2: Figure S2). The up-regulation of T-UC.324 was confirmed in all three cell lines. Levels of T-UC.300A could not be detected by qPCR in SK-N-BE, however its downregulation was validated in both SH-SY5Y and LAN-5. The intergenic T-UCRs (9/32) can be considered independent transcripts as they are not embedded in protein coding host genes. Among the intragenic T-UCRs, 10 were transcribed in the opposite direction to their host gene, and therefore also represent independently transcribed ncRNAs. For the partially exonic or intronic T-
UCRs that were expressed in a sense direction with their host gene, at least 3 likely represent independent transcripts as their level of expression is not significantly correlated with the host exon expression (e.g. host gene down-regulated in response to ATRA while T-UCR is up-regulated and vice versa). The correlation of T-UCR /host gene expression could only be carried out for intragenic T-UCRs that had host gene exons within their surrounding tiled regions (2500 bp upstream and $500 \mathrm{bp}$ downstream of each UCR). Exonic T-UCRs expressed in the sense direction relative to their host gene had the highest correlations with their host genes (median correlation $=0.68)$, with $67 \%$ showing a significant correlation $(\mathrm{p}<0.05)$. Intronic T-UCRs expressed anti-sense to their host gene displayed the lowest correlation with their host genes (median correlation $=0.13$ ), with just $12 \%$ showing a significant correlation $(\mathrm{p}<0.05)$ (Figure 2A; Additional file 3: Table S1). In summary, 22 independent non-coding transcriptional units that were responsive to ATRA could be identified, while 5 ATRA responsive intronic or exonic T-UCRs were identified whose expression was significantly correlated with their host genes. Consistent with these findings, some of the T-UCR host genes, such as HOXC4, are known to be responsive to ATRA [22].

\section{Investigation of regulation through transcriptional interference}

Independent intragenic T-UCRs, either sense or anti-sense, could be involved in the regulation of their host genes through different types of transcriptional interference where transcriptional elongation has a direct, cis-acting suppressive effect on a second transcriptional process [23]. Analysis of all intragenic T-UCRs and their host gene expression revealed seven T-UCRs whose expression was significantly anti-correlated with that of their host gene (Additional file 3: Table S1). None of the seven T-UCRs showed a consistent change in expression across cell lines following ATRA-treatment, however in individual samples T-UCR expression was significantly anti-correlated with host gene expression $(\mathrm{p}<0.05)$ which might suggest a form of regulation through transcriptional interference. The anti-correlation between T-UC.350 and T-UC.90 and their host genes can be seen in Figure 2B, C. Of particular interest is the T-UC.350/DACH1 anti-correlation. DACH1 is a tumor suppressor gene, whose expression is reduced in prostate and endometrial cancer correlating with increased tumor progression and invasion [24,25]. The significant anti-correlation seen here between the two transcripts $(\mathrm{p}<0.05)$ suggests a possible cis regulation between the two in different cancer types.

siRNA knockdown and functional analysis of t-uc.300a The prioritization of differentially expressed T-UCRs for functional assessment was based on the statistical 


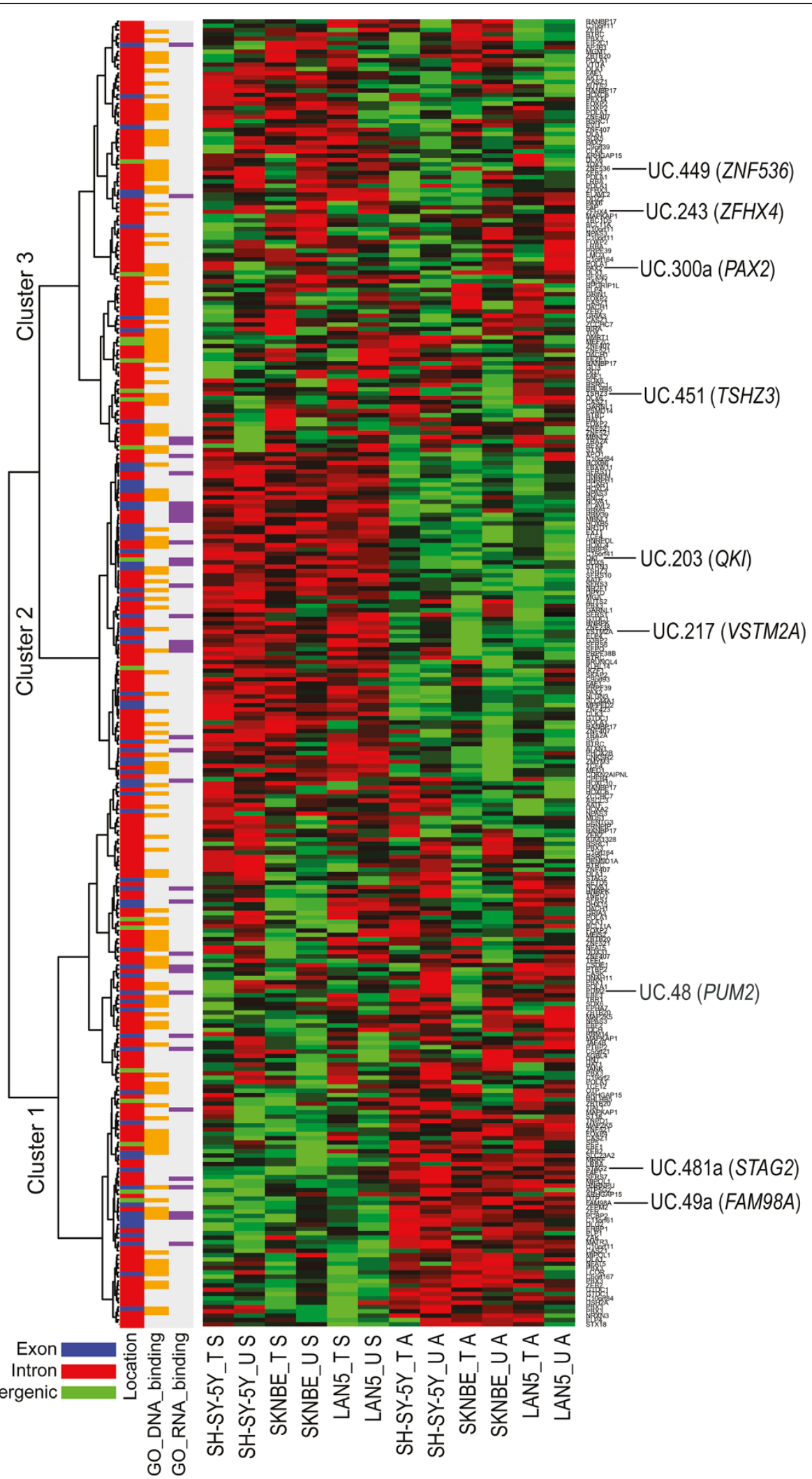

Figure 4 (See legend on next page.) 
(See figure on previous page.)

Figure 4 Unsupervised analysis of intragenic T-UCR cell line expression profiles. Subgroups of T-UCRs are expressed in three main clusters: Cluster 1, T-UCRs that are expressed anti-sense to the host gene expression; Cluster 2, T-UCRs that are expressed sense to host gene expression; Cluster 3, T-UCRs that are expressed both sense and anti-sense to host gene expression and show a significant over-representation of DNA binding proteins within their host genes relative to the other two clusters. Intragenic T-UCRs that are altered by ATRA are indicated with their host gene on the right side of the heatmap.

significance of differential expression between untreated and ATRA-treated cells, in addition to other features of interest such as proximity to cancer-associated genomic regions or deregulation in other cancer types. The transcript of most interest from our list was T-UC.300A, which is down-regulated following ATRA treatment (Table 1). This UCR is located within a frequent loss of heterozygosity $(\mathrm{LOH})$ region on chromosome arm 10q, anti-sense to the developmental gene $P A X 2$, a gene whose under- or over-expression is associated with both pediatric and adult kidney pathology [26]. In addition, TUC.300A levels were previously shown as up-regulated in colorectal carcinoma tumors [13]. Considering its down-regulation in ATRA-treated neuroblastoma cells, this suggests that T-UC.300A could play a role in both pediatric and adult cancers. T-UC.324 was selected as our second T-UCR for functional analysis. The most significantly up-regulated transcript across the three ATRA-treated cell lines $(\mathrm{p}=0.002)$ (Table 1, Figure 1), this ncRNA is expressed anti-sense to the Mpedd2 gene, which displays an anti-tumorigenic function in differentiated NB cells and is also highly expressed [27]. Both T-UC.324 and T-UC.300A are independent transcripts, and not transcribed as part of a host gene.

SH-SY5Y and LAN-5 cells were transfected with custom designed siRNAs against either T-UC.324 or T-UC.300A to knock down these transcripts and assess any effect of this knockdown on cell invasion and proliferation rates. Knockdown of T-UC.324 abrogated its ATRA-induced up-regulation by $40 \%$ to $60 \%$ (Figure $3 \mathrm{~A}$ ) and effected a small but significant relief from the decrease in cell proliferation induced by ATRA $(\mathrm{p}<0.05)$ (Figure 3B). Although this effect was statistically significant across biological repeats, it was minimal and considered unlikely to contribute to any major biological role. However, knockdown of T-UC.300A resulted in a more significant decrease $(\mathrm{p}<0.01)$ in cell viability (Figure $3 \mathrm{C}, \mathrm{D})$. Using the Cell Proliferation ELISA, BrdU (colorimetric) kit (Roche), we determined that this decrease in cell viability was the result of a down-regulation in cell proliferation (Figure 3E), as opposed to an increase in apoptosis (Figure 3G). In addition, knockdown of the T-UC.300A transcript resulted in a decrease in cell invasion $(\mathrm{p}=0.008)$ (Figure $3 \mathrm{~F})$. There was no obvious change in cell morphology following knockdown. As T-UC.300A is significantly down-regulated following ATRA treatment, this reduction in both proliferation and invasion rates seen with siRNA knockdown supports a role for this transcript in the inhibition of differentiation, and as a promoter of proliferation and invasion.

Gene expression microarray analysis of SH-SY5Y cells following siRNA knockdown of T-UC.300A ( $n=2)$ was carried out in an effort to identify any protein-coding genes which may be involved in T-UC.300A promotion of proliferation and invasion, or inhibition of differentiation prior to ATRA treatment. Following siRNA knockdown of T-UC.300A, 150 genes were up-regulated and 123 genes were down-regulated (at least 1.5 fold in both biological repeats). The genes with the greatest mean fold change are listed in Table 2. qPCR was carried out on COL1A2 to validate array results (Additional file 4: Figure S3). By comparing the differentially expressed genes with genes we have previously shown to be altered by ATRA in SH-SH5Y cells $(n=2)$ [19], we found that 46 out of the 150 genes up-regulated following T-UC.300A knockdown were also up-regulated following ATRA treatment (at least 1.5 fold, $\mathrm{n}=2$ ). In addition, 23 genes down-regulated following siRNA knockdown were also down-regulated following ATRA treatment. These results suggest that the altered expression of these genes following ATRA could be the result of changing T-UC.300A levels.

\section{Unsupervised analysis of T-UCR expression}

In order to determine if T-UCRs had sub-groupings with characteristic expression behaviour, unsupervised analysis was performed on the intragenic T-UCR cell line expression profiles. The T-UCR data was pre-processed as described in the Methods section.

From the heatmap in Figure 4, we see that sub-groups of intragenic T-UCRs are expressed in three main clusters. Cluster 1 and Cluster 2 represent T-UCRs that are expressed in the anti-sense and sense direction, respectively, relative to the host gene transcription in a manner largely independent of cell line or treatment. Another subset (Cluster 3 ) is expressed in a more variable fashion over samples and treatments. This latter subset is significantly enriched for DNA binding proteins $(P=2.9 \mathrm{e}-02)$ and has a significant under-representation of both RNA binding proteins $(P=6.6 \mathrm{e}-04)$ and of exonic T-UCRs $(P=6.0 \mathrm{e}-06)$. This suggests that certain sub-groups (present in Clusters 1 and 2) T-UCRs may be transcribed exclusively in a either a sense or anti-sense direction relative to the host gene whereas another subset $\mathrm{T}$ - 
UCRs (present in Cluster 3) may be transcribed in both directions relative to host gene.

\section{Discussion}

This study represents the first profiling and functional analysis of transcribed long ultra-conserved ncRNAs that are differentially expressed during ATRA induced differentiation of NB cell lines. Although their 100\% homology between human, mouse and rat denotes possible critical roles in vertebrate development, there has been limited investigation of their roles in cancer development $[13-15,28,29]$. Here we sought to identify and investigate the functionality of T-UCRs differentially expressed in NB cell lines following ATRA-treatment. We have identified a role for T-UC.300A in the promotion of proliferation and invasion of NB cells prior to ATRA-induced differentiation. This intronic transcript is anti-sense to the developmental transcription factor PAX2, a gene whose function is essential during embryonic development and morphogenesis but whose overexpression also contributes to the pathogenesis of diseases such as renal cell carcinoma and medulloblastoma [30,31]. There is an emerging role for ncRNAs in the regulation of HOX genes both in cis, through the process of transcription itself, and in trans through a direct role in gene regulation such as chromatin re-modelling [32,33]. The repressive role of T-UC.300A was alleviated by siRNA knockdown arguing against a role for transcriptional interference and, indicating that this ncRNA most likely exerts its effect in trans. PAX2 expression was not detected in either ATRA-treated or untreated cells, also supporting a trans-acting role for T-UC.300A.

Gene expression microarray analysis showed that 150 genes were up-regulated following T-UC.300A knockdown, 46 of which were also up-regulated by ATRA treatment. The gene with the greatest increase in expression, INPP5D, encodes the SH2-containing inositol phosphatase 1 (SHIP1), a negative regulator of the PI3K/ AKT pathway. Gene transfer of INPP5D has been shown to reduce proliferation in $\mathrm{CD} 34+$ cells from patients with acute myeloid leukemia (AML) [34]. A number of the commonly differentially expressed genes are of particular interest in identifying a putative pathway for TUC.300A function. At least two of the most highly upregulated genes (CALCA, KLF2) have been reported as hypermethylated in a number of cancers, including neuroblastoma [35-38]. In addition, KLF2 is involved in the inhibition of proliferation and migration and is silenced in cancer by the Polycomb repressive complex 2 protein EZH2 $[39,40]$. Furthermore KLF2 expression in acute promyelocytic leukemia (APL) can be restored by ATRA treatment [41]. A recent study has shown that overexpression of the long ncRNA HOTAIR altered PRC2 occupancy in breast cancer cells [42]. With this in mind, it is possible that the corresponding up-regulation of genes such as KLF2, CALCA and COL1A2 following knockdown of T-UC.300A could be indicative of a role for this T-UCR in chromatin re-programming possibly by targeting methylation-associated complexes such as PRC2. Therefore reduced T-UC.300A levels (either by siRNA or ATRA treatment) could co-ordinate the derepression of these genes by reducing methylation at their promoters and explain the observed increase in gene expression.

Our results show that the expression of a number of T-UCRs is altered by ATRA-induced differentiation. However it is clear that a number of mechanisms are at work in their regulation, and consequently in the downstream regulation of T-UCR targets themselves.

\section{Additional files}

\begin{abstract}
Additional file 1: Figure S1. Untreated and ATRA-treated SK-N-BE cells. Cells were treated with ATRA ( $5 \mu \mathrm{M}$ final concentration) daily for 7 days. (A) Cells were probed using the neuronal marker BIII Tubulin (Abcam), and were incubated with the flourescein-conjugated goat anti-rabbit Alexa Flour 488 antibody (Invitrogen). (B) Western Blot for Blll-Tubulin, MYCN and a-Tubulin (loading control) in untreated and ATRA-treated cells.

Additional file 2: Figure S2. qPCR validation of tiling array results. Array results were validated for T-UC.324 and T-UC.300A by qPCR using gene-specific RT primers and custom-designed TaqMan Assays. Error bars represent standard deviation from the mean across at least two biological repeats.

Additional file 3: Table S1. Correlation of intragenic T-UCR and host gene expression. T-UCRs are in four groups: exonic sense; exonic antisense; intronic sense; intronic anti-sense. Shaded cells denote significance or approaching significance.

Additional file 4: Figure S3. qPCR validation of gene expression results. Gene expression microarray results were validated by qPCR for COL1A2 in SHSY5Y cells following knockdown of T-UC.300A. Figure shows results from array and from $\mathrm{gPCR}$ analysis. Error bars represent standard deviation from the mean across two biological repeats.
\end{abstract}

\section{Competing interests}

The authors declare that they have no competing interests.

\section{Authors' contributions}

KMW carried out laboratory work pertaining to this study, participated in its design and drafted the final manuscript. KB carried out bioinformatic analyses. NHF carried out laboratory work. RLS conceived the study, and participated in its design and co-ordination and helped to draft the manuscript. All authors read and approved the final manuscript.

\section{Acknowledgements}

This work was supported in part by grants from Cancer Research Ireland and the Children's Medical and Research Foundation.

Received: 13 August 2012 Accepted: 20 March 2013

Published: 8 April 2013

\section{References}

1. Brodeur GM: Neuroblastoma: biological insights into a clinical enigma. Nat Rev Cancer 2003, 3(3):203-216.

2. De Preter $K$, et al: Human fetal neuroblast and neuroblastoma transcriptome analysis confirms neuroblast origin and highlights neuroblastoma candidate genes. Genome Bio/ 2006, 7(9):R84. 
3. McArdle $L$, et al: Oligonucleotide microarray analysis of gene expression in neuroblastoma displaying loss of chromosome 11q. Carcinogenesis 2004, 25(9):1599-1609.

4. Wang $Q$, et al: Integrative genomics identifies distinct molecular classes of neuroblastoma and shows that multiple genes are targeted by regional alterations in DNA copy number. Cancer Res 2006, 66(12):6050-6062.

5. Oberthuer A, et al: Customized oligonucleotide microarray gene expression-based classification of neuroblastoma patients outperforms current clinical risk stratification. J Clin Oncol 2006, 24(31):5070-5078.

6. Vermeulen J, et al: Predicting outcomes for children with neuroblastoma using a multigene-expression signature: a retrospective SIOPEN/COG/ GPOH study. Lancet Oncol 2009, 10(7):663-671.

7. Bray l, et al: Widespread dysregulation of MiRNAs by MYCN amplification and chromosomal imbalances in neuroblastoma: association of miRNA expression with survival. PLoS One 2009, 4(11):e7850.

8. Buckley $P G$, et al: Chromosomal and microRNA expression patterns reveal biologically distinct subgroups of 11q- neuroblastoma. Clin Cancer Res 2010, 16(11):2971-2978.

9. Chen Y, Stallings RL: Differential patterns of microRNA expression in neuroblastoma are correlated with prognosis, differentiation, and apoptosis. Cancer Res 2007, 67(3):976-983.

10. Mestdagh $P$, et al: MYCN/c-MYC-induced microRNAs repress coding gene networks associated with poor outcome in MYCN/c-MYC-activated tumors. Oncogene 2010, 29(9):1394-1404.

11. Schulte $\mathrm{JH}$, et al: Deep sequencing reveals differential expression of microRNAs in favorable versus unfavorable neuroblastoma. Nucleic Acids Res 2010, 38(17):5919-5928.

12. Bejerano $\mathrm{G}$, et al: Ultraconserved elements in the human genome. Science 2004, 304(5675):1321-1325.

13. Calin GA, et al: Ultraconserved regions encoding ncRNAs are altered in human leukemias and carcinomas. Cancer Cell 2007, 12(3):215-229.

14. Mestdagh $P$, et al: An integrative genomics screen uncovers ncRNA T-UCR functions in neuroblastoma tumours. Oncogene 2010, 29(24):3583-3592.

15. Scaruffi $P$, et al: Transcribed-Ultra Conserved Region expression is associated with outcome in high-risk neuroblastoma. BMC Cancer 2009, 9:441

16. Pahlman S, et al: Retinoic acid-induced differentiation of cultured human neuroblastoma cells: a comparison with phorbolester-induced differentiation. Cell Differ 1984, 14(2):135-144.

17. Wagner LM, Danks MK: New therapeutic targets for the treatment of high-risk neuroblastoma. J Cell Biochem 2009, 107(1):46-57.

18. Thiele CJ, Reynolds CP, Israel MA: Decreased expression of N-myc precedes retinoic acid-induced morphological differentiation of human neuroblastoma. Nature 1985, 313(6001):404-406.

19. Das $\mathrm{S}$, et al: MicroRNA mediates DNA demethylation events triggered by retinoic acid during neuroblastoma cell differentiation. Cancer Res 2010, 70(20):7874-7881.

20. Bolstad BM, et al: A comparison of normalization methods for high density oligonucleotide array data based on variance and bias. Bioinformatics 2003, 19(2):185-193.

21. Irizarry RA, et al: Exploration, normalization, and summaries of high density oligonucleotide array probe level data. Biostatistics 2003 4(2):249-264.

22. Kim DY, et al: Up-regulated hoxC4 induces CD14 expression during the differentiation of acute promyelocytic leukemia cells. Leuk Lymphoma 2005, 46(7):1061-1066

23. Callen BP, et al: Transcriptional interference between convergent promoters caused by elongation over the promoter. Mol Cell 2004, 14(5):647-656.

24. Wu K, et al: The cell fate determination factor dachshund inhibits androgen receptor signaling and prostate cancer cellular growth. Cancer Res 2009, 69(8):3347-3355.

25. Nan F, et al: Altered expression of DACH1 and cyclin D1 in endometrial cancer. Cancer Biol Ther 2009, 8(16):1534-1539.

26. Harshman LA, Brophy PD: PAX2 in human kidney malformations and disease. Pediatr Nephrol 2011

27. Liguori L, et al: The metallophosphodiesterase Mpped2 impairs tumorigenesis in neuroblastoma. Cell Cycle 2012, 11(3):569-81.

28. Braconi $\mathrm{C}$, et al: Expression and functional role of a transcribed noncoding RNA with an ultraconserved element in hepatocellular carcinoma. Proc Natl Acad Sci USA 2011, 108(2):786-91.
29. Sana J, et al: Expression levels of transcribed ultraconserved regions uc.73 and uc.388 are altered in colorectal cancer. Oncology 2012, 82(2):114-8

30. Gruss P, Walther C: Pax in development. Cell 1992, 69(5):719-22.

31. Gnarra JR, Dressler GR: Expression of Pax-2 in human renal cell carcinoma and growth inhibition by anti-sense oligonucleotides. Cancer Res 1995 55(18):4092-8

32. Sessa $L$, et al: Noncoding RNA synthesis and loss of Polycomb group repression accompanies the colinear activation of the human HOXA cluster. RNA 2007, 13(2):223-39.

33. Rinn $\mathrm{L}$, et al: Functional demarcation of active and silent chromatin domains in human HOX loci by noncoding RNAs. Cell 2007, 129(7):1311-23.

34. Metzner A, et al: Reduced proliferation of CD34(+) cells from patients with acute myeloid leukemia after gene transfer of INPP5D. Gene Ther 2009, 16(4):570-3.

35. Yao D, et al: Quantitative assessment of gene methylation and their impact on clinical outcome in gastric cancer. Clin Chim Acta 2012, 413(7-8):787-94.

36. Olk-Batz C, et al: Aberrant DNA methylation characterizes juvenile myelomonocytic leukemia with poor outcome. Blood 2011, 117(18):4871-80.

37. Caren $\mathrm{H}$, et al: Identification of epigenetically regulated genes that predict patient outcome in neuroblastoma. BMC Cancer 2011, 11:66.

38. Moran A, et al: Methylation profiling in non-small cell lung cancer: clinical implications. Int J Oncol 2012, 40(3):739-46.

39. Taniguchi $\mathrm{H}$, et al: Silencing of Kruppel-like factor 2 by the histone methyltransferase EZH2 in human cancer. Oncogene 2012, 31(15):1988-94.

40. Black AR, Black JD, Azizkhan-Clifford J: Sp1 and kruppel-like factor family of transcription factors in cell growth regulation and cancer. J Cell Physiol 2001, 188(2):143-60

41. Humbert $M$, et al: Deregulated expression of Kruppel-like factors in acute myeloid leukemia. Leuk Res 2011, 35(7):909-13.

42. Gupta RA, et al: Long non-coding RNA HOTAIR reprograms chromatin state to promote cancer metastasis. Nature 2010, 464(7291):1071-6.

doi:10.1186/1471-2407-13-184

Cite this article as: Watters et al: Expressional alterations in functional ultra-conserved non-coding rnas in response to all-trans retinoic acid induced differentiation in neuroblastoma cells. BMC Cancer 2013 13:184.

\section{Submit your next manuscript to BioMed Central and take full advantage of:}

- Convenient online submission

- Thorough peer review

- No space constraints or color figure charges

- Immediate publication on acceptance

- Inclusion in PubMed, CAS, Scopus and Google Scholar

- Research which is freely available for redistribution 